\title{
Proposed Clinical Diagnostic Records for Dental Implant
}

\author{
Hassan H Koshak* \\ Periodontist and Implantologist, Saudi Arabia
}

Submission: September 04, 2017; Published: September 11, 2017

*Corresponding author: Hassan H Koshak, Head of the Dental Department and Dental Educator at Comprehensive Specialized Polyclinic, Ministry of Interior Security Forces Medical Services, Jeddah, Kingdom of Saudi Arabia, P.O. Box 108999, Jeddah 21352, KSA, Tel: +966 (0) 555507035; Email: Koshak.hh@gmail.com

\section{Introduction}

Dental Record is documents related to medical and dental history, clinical examination findings, diagnosis, prognosis and performed treatment steps [1]. It prevents confusion resulting from treatment performed by deferent operators [2]. Images, photographs, study cast and $\mathrm{x}$ ray findings should be included in the dental records [3]. Written consent of the patient is an integral part of any record [4]. The dental record is important because it may be used in a court of law to establish the diagnostic information that was obtained and the treatment that was rendered to the patient. Detailed records should be kept regarding the treatment procedures, including any difficulties encountered, subsequent actions taken, and the final outcome of the treatment. According to Health and Social Care Act, up to 2004 [5] only two reports mentioned the quality of record keeping. So, the present study was performed as a trail the quality of dental records of Riyadh Colleges of Dental and Pharmacy (RCsDP).

\section{Materials and Methods}

\section{Study Design}

In the first part of the current research the followings were done:

The file currently in use in RCsDP that was performed following directions of American Dental Association (ADA), American Academy of Periodontology (AAP), and Association of State and Territorial Dental Directors.

Some modifications were done to the dental records according to (Leven and Alman, 2000), and to what have been recently reported in literature. A new file was proposed. The proposed forms were sent for reviewers for evaluation. Reviewers were: oral and maxillofacial surgeons, oral diagnosis consultants, radiologists, and dental practitioners. Reviewers were from faculty members of RCsDP, and ministry of health hospital in Riyadh. The proposed documents were sent to reviewers asking them to comment on the following items concerning the file:
a) Time needed to complete.
b) Coverage of all needed necessary information.
c) Clarity of all items to patient.
d) If there is any non-relevant information. Please specify.
e) If there is any missing item, feel free to add.

The comments from reviewers were studied and classified into suggestions related to design, history, examination findings and treatment plan.

\section{Statistical Analysis}

Statistical analysis was done for the received comments. Test of agreement was used according to Sax, 2010. The amount of agreement was calculated by counting the proportion of times raters (reviewer) agreement on specific item. Reliability test was done and the rater was considered unreliable when two rating were different for the same item. According to the result of test of agreement, modifications were done for the clinical diagnostic chart and consent form. Comments from patients were also statistically analyzed using test of improvement. Records were adjusted according to reviewer comments and was disrupted again and the same procedure repeated.

\section{Results}

Statistestical analysis for comments received from reviewers. The reliability test excludes only four raters from the study who were considered unreliable.

Analysis of reviewer comments revealed the followings:

a) Six percent of reviewer advised to change title for personal data to base line data rather than patient information. 
b) Dental History: three percent recommended detailed description of parafunctional habits.

c) Medical history: the proposed change was to put it in the form of checklist and allow area for updating it for easier identification of any recent changes

d) Clinical examination comments: percentage recommended adding smile analysis, and specific criteria concerning maxillary-mandibular relationship. Twenty nine percent recommended eliminating periodontal screening records (PSR), while 9\% advised to modify the format of dental radiographic interpretation to allow more specific diagnosis

Also, a more detailed description of the implant placement surgery was added to consent form Figure 1. Analysis of patient's consent comments related to proposed patient consent revealed the followings:

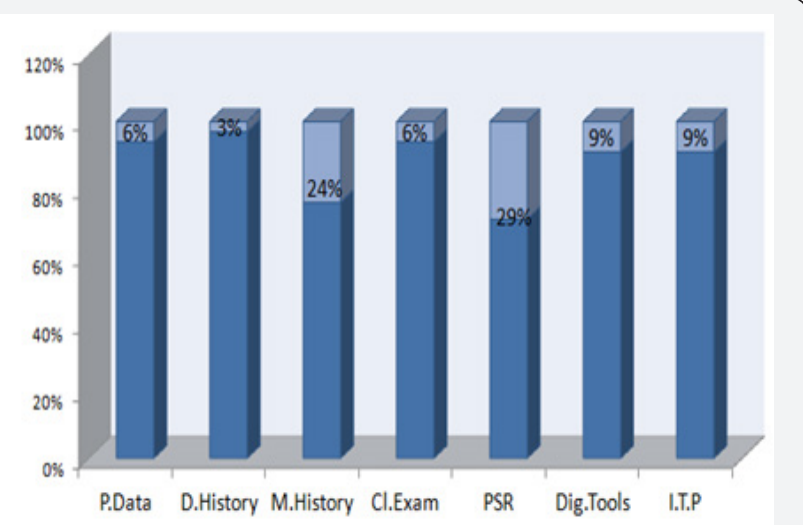

Figure 1: Reviewer comments regarding proposed changes in dental records.

a. Comments regarding introduction were eighteen percent

\section{b. Changes according to literature review findings:}

Some instructions were added to the consent form that is essential for decreasing risks of post implant failure Figure 2.

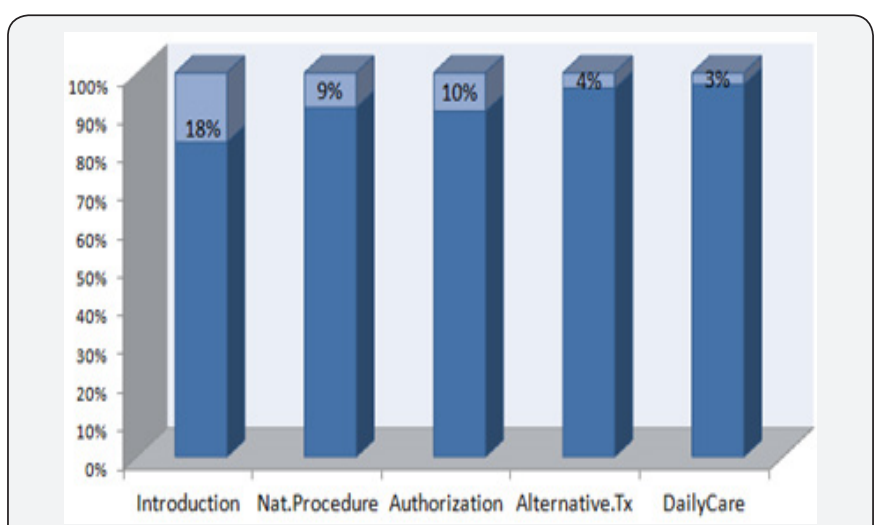

Figure 2: Comments regarding the primary consent form. c. Comment regarding the primary post surgical instruction form: -

I. Comments regarding presentation and outline:

There were no comments regarding presentation of the form, all agreed and recommend keeping as it is. Second assessment of the proposed dental records: There were no additional comments from the reviewers regarding the three tested forms in the second evaluation. Results of the patients' comments regarding the consent form: Test of improvement for the patient opinion regarding proposed consent revealed the followings:

i. Percentage of acceptance changed from seventy eight percent to ninety one percent.

ii. Clarity of the form improved from nine percent to four percent.

iii. Eight percent considered the information of the form frightening, after modifications ratio became three percent.

iv. The five percent that considered the form unclear, was reduced to two percent after modification.

II. First and Second patient Comments regarding the consent form (Figure 3):

\begin{tabular}{|l|c|}
\hline Acceptable & $78 \%$ \\
\hline Vague & $9 \%$ \\
\hline Frightening & $8 \%$ \\
\hline Un acceptable & $5 \%$ \\
\hline & \\
\hline Acceptable & $91 \%$ \\
\hline Vague & $4 \%$ \\
\hline Frightening & $3 \%$ \\
\hline Un acceptable & $2 \%$ \\
\hline \\
Figure 3: First and Second patient Comments regarding the \\
consent form.
\end{tabular}

\begin{tabular}{|c|c|c|c|}
\hline Opinion & $\%$ Pre & $\%$ Post & P.valu \\
\hline Acceptable & $78 \%$ & $91 \%$ & 0.0098 \\
\hline Vague & $9 \%$ & $4 \%$ & 0.149 \\
\hline Frightening & $8 \%$ & $3 \%$ & 0.119 \\
\hline Un acceptable & $5 \%$ & $2 \%$ & 0.247 \\
\hline \multicolumn{4}{|c|}{$\begin{array}{l}\text { Figure 4: Proportion } t-\text { test } \alpha=0.05, P \text { value }=0.0098, P \\
\text { value }=0.0098<0.05 \text {-Shows that it is significant. } P \text { The result is } \\
\text { acceptable. }\end{array}$} \\
\hline
\end{tabular}




\section{Global Journal of Otolaryngology}

Test of improvement for the patient opinion regarding proposed consent form (Figure 4):

\section{Discussion}

The present study was conducted to optimize an accurate, and comprehensive patient records for each patient treated in clinics of RCsDP. The standard for this clinical record was planned to be obtained according to what is reported in evidence based literature review. Plus what had been obtained from reviewers that judged the proposed records. In the present study, the detailed medical history was kept the same but it was divided into two pages. This was done in order not to be condensed and to allow for updating in the same page. Updating of medical history in the same area will allow easier identification of any new disease or condition. According to American Academy of Pediatrics, it should be performed in each dental visit [1].

Regarding reviewers comment concerning difficulty for patients to answer questions of the medical history section by themselves, a separate screening form was designed to be answered by the patient. While for the diagnostic dental chart it will be the responsibility of operating dentist. This was done according to ADA report, 2010, [2] which stated that the dentist is ultimately responsible for the patient's chart. In the section related to personal information of the diagnostic chart, detailed information regarding intensity of smoking was added to both personal information and consent form. This was done according to literature documents that discussed this topic.

Leo, [3] reported that high percentages of smokers visit dentist every year which makes smoking cessation part of the role of dentists. This will decrease possibility of complications after invasive dental procedures on the short term and oral and periodontal health on the long term. Study by Schwartz-Arad et al 2002 [3] concluded that smokers have twice risk for developing implant failure than non smokers specially with bone height less than $4 \mathrm{~mm}$. Implant survival may be increased in smokers by implementing smoking cessation programs. There is conflicting and scanty data available as to the appropriate length of time that would be required to elicit a positive effect on implant outcomes with studies having a range of 1 to 8 weeks of smoking cessation. If this recommendation was adopted as a guideline for patient selection, we would be relying on patient self-reporting, which is notoriously unreliable [4].

In the present study, information regarding diagnosis of Parafunctional habits that may have detrimental effect on implant survival was incorporated in the file. Larking 2004 [5] stated that very high force result from bruxism, could contribute to implant failure. The evidence that Parafunction contributes to implant failure is only modest, depends on supplementary analyses of studies investigating other factor even then. There are a few practical guidelines as to minimize the chance of implant failure. Besides the recommendation to reduce or eliminate bruxism itself, these guidelines concern the number and dimensions of the implants, the design of the occlusion and articulation patterns, and the protection of the final result with a hard occlusal stabilization splint [6].

The highest percentage of reviewers' comments (29\%) were suggesting removal of PSR from periodontal examination. Accordingly it was removed from the chart. This was supported from literature by Landry and Jean 2002 [7] who reported PSR accelerate the screening of periodontal disease but it may under or over estimate existing periodontal conditions. Also, Hassel and Wolf.2006 stated that PSR provide indications as weather additional and more detailed examination are necessary. In the final form of diagnostic chart, periodontal examination was emphasized. Reevaluation measurement for pocket depths, plaque and gingival indices were incorporated in a comparable way with the first examination findings. This was done in response to literature review findings that point out to the strong relationship between periodontal diseases and implant failure Simonis et al, 2010 [8].

Since a variety of clinical and radiographic parameters have been identified to predict long term success of dental implant, the following items were added to the examination section of dental record. They include biologic width, papilla and soft tissue height, soft tissue volume, and amount of keratinized gingiva and biotype of mucosa [9]. The radiographic interpretation areas of the studied dental record were modified by adding the use of Cone Beam Computer Tomography Scanning Technology. This was done according to the fact that two-dimensional film images have been found to have limitations because of inherent distortion factors, little information regarding bone density, bone width, or proximity to vital structures [10].

Severity of dental implant case was added to the surgery section of the diagnostic chart. This was done according to guidelines for the Provision of Dental Implants of the College of Dental Surgeons of Hong Kong [11]. This classification specify how will do implant procedure according to degree of severity. That is why oral maxillofacial surgeon was replaced by the title "operator" in consent form considering the technical or biological difficulties about dental implant as a treatment option at an individual level. A patient's informed consent is a fundamental aspect of the proper provision of dental care. Improvement of the informed consent is important to create more realistic expectations of the patient. So objections of patients to the proposed consent form and describing its statements as frightening was not taken into consideration in the present study. This decision was supported by the 2015 Supreme Court ruling in the obstetric case of Montgomery V Lanark shire Health Board [12]. Also, stated that a clinician has the responsibility to ensure that individuals genuinely understand all procedure risks and benefits as well as available alternatives [13].

Automated administrative databases are omnipresent in medical and dental health, care. These automated databases are 
now commonly linked to data extracted from electronic medical record and are used to measure health system performance, adjust payment, access providers for quality improvement purposes and inform policy analyses [14]. Such data sources can be used to perform case control and longitudinal observation studies [15]. Electronic patient records for dentistry using criteria are available in two documents produced by the American Dental Association 1999 and 2000 [16]. The proposed dental records can serve as a base for preparation and transforming clinic paper documents into electronic ones. This will be more predictable after accreditation and updating of these records [17-24].

\section{Conclusion}

The patient records should be reviewed and updated regularly. Frequent audit checking for patient records is essential for ultimate patient care.

\section{References}

1. American Academy of Pediatric dentistry (2015) Clinical records guidelines. Guidelines on record keeping.

2. American Dental Association (2010) ADA Accredited standards committee MD156 task group on dental informatics. ADA technical reports No 1004. Computer software performance for dental practice software. Chicago, USA.

3. Schwartz-Arad D, Naama S, Nachum, Mamlider A (2002) Smoking and Complications of Endosseous Dental Implants. J Periodontol 73: 153157.

4. Leichter J, Lyons K (2004) Dental Implants: Patient Selection Factors Evidence-Based Review. Healthcare Team.

5. Larking P, Leichter J, Lyons K (2004) Dental Implants: Patient Selection, Patient Satisfaction and Cost Factors. Evidence Based Healthcare Advisory Group.

6. Lobbezoo F, Zaag JD, Naeije M (2006) Bruxism its multiple causes and its effects on dental implant an updated review. Journal of Oral Rehabilitation 33: 293-300.

7. Landry RG, Jean M (2002) Periodontal Screening and recording (PSR) index, int dent j.1.

8. Simonis P, Dufour T, Tenenbaum H (2010) Long-term implant survival and success: a 10-16- year follow-up of non-submerged dental implants. Clinical Oral Implants Research 21: 772-777.

9. Benic GI, Wollebk, Sancho-Punchades M, Hammerle CH (2012) Systematic review of parameters and methods for professional assessment of aesthetics in dental implant research. J of clin periodontal. 39: 160-192.

10. Scott D, Ganz (2008) Computer-aided design/ computer aided manufacturing applications using CT and Cone Beam CT scanning technology. Dent clin N Am 52: 777-808.

11. David K (2005) Medico-legal aspects of dental implants. Hong Kong Dental Journal 2: 54-46.

12. Montogomery (Appellant) V Lanar Kshire Health Board (Respondent) (Scotland), 2015 UKSC 11.

13. Suetife (2016) What's new in the process of consent? Refereed paper. SAAD DIGEST 32.

14. Peabodoyjw, Luck J, Jaains, Bertentthal D, Passman GP (2004) Assessing the Accuracy of Administrative Data in Health Information System. Med Car 42: 1066-1072.

15. Spangler, Chaudhari M , Barlow W, Newton KM, Inge R, et al. (2012) Using Administrative Data For Epidemiological Research: Case Study To Identify Person With Periodontitis. Periodontology 2000(58): 143152.

16. American Dental Association (2000) ADA standards committee on dental informatics. ANS/ADA specification No1000 for standard clinical data artitechure for the structure and content of an electronic health record, Chicago, USA.

17. Leo F A Stassen (2015) Smoking cessation and the role of dental practitioner. Peer reviewed. Journal of Irish Dental Association 61: 9092.

18. Charangowda BK (2010) Dental records: An overview. J Forensic Dent Sci 2(1): 5-10.

19. Madhusudan Astekar, Swati Saawarn, Nisheeth Saawarn (2011) Maintaining dental records: Are we ready for forensic needs. Journal of Forensic Dental Sciences 3(2): 52-57.

20. Dental Record Keeping Guidelines, April 2013, College of Dental surgeons of British Columbia, UK.

21. Health DO (2008) Health and Social Care Act. Department of Health London, UK.

22. Crawford J, T Beresford, K Lafferty (2001) The CRABEL score - a method for auditing medical records. Annals of The Royal College of Surgeons of England 83(1): 65.

23. Chrcanovic BR, Albrektsson T, Wennerberg A (2014) Reasons for failures of oral implants. J Oral Rehabil 41: 443-476.

24. Choukroun Joseph, Georges Khoury, Fouad Khoury (2014) Two Neglected Biologic Risk Factors in Bone Grafting and Inplantology: High Low-Density Lipoprotein Cholesterol and low serum vitamin D. Journal of Oral Implantology 40: 1.
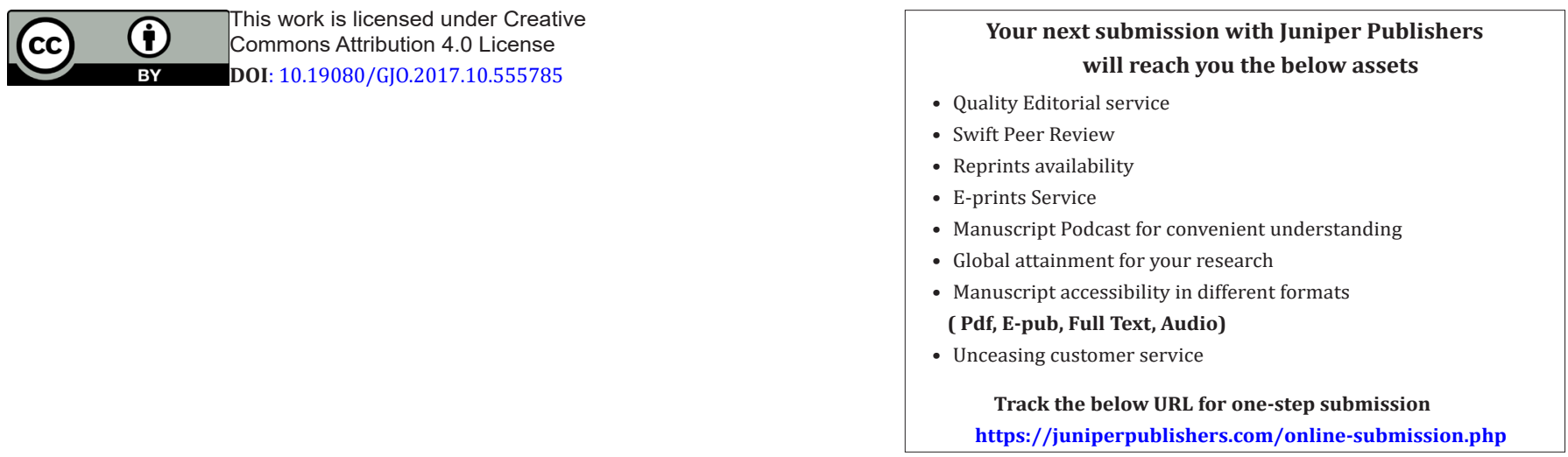